\title{
Non-constructive Proofs of a Metamathematical Theorem Concerning the Consistency of Analysis and its Extension
}

\author{
By Shôji Maehara \\ Waseda University, Tokyo, \\ Toshio Nishimura \\ Hōsei University, Tokyo, \\ and \\ Setsuya Seri \\ St. Paul's University, Tokyo
}

In this paper we shall treat the following theorem, and to which we shall give three proofs :

Theorem. Let $A$ be a formula in the predicate calculus of 1 st order. If $A$ is provable in the type-logic, that is, in the predicate calculus of $\omega$ th order, then it it also provable in the predicate calculus of 1 st order.

First, S. Seki obtained a proof of the theorem by applying Gödel's completeness theorem [1]. In the next, S. Maehara obtained the second proof by using the so-called Lindenbaum algebra [2]. And, T. Nishimura obtained the third proof and an extension of the theorem by utilizing Schütte's idea [3].

This theorem means: If a system of axioms is consistent in the predicate calculus of 1st order, then it is also consistent in the type logic. On the other hand, it is well-known that, in the predicate calculus of 1st order, the natural number theory of some kind is consistent [4]. Therefore, our theorem implies that a considerably large part of analysis is also consistent. But it should be remarked that none of our proofs is finitary. 
Prof. G. Takeuti extended Gentzen's $L K$ [5] to a type-logic, and called his system $G L C$. He calls the next statement the fundamental conjecture of $G \cdot L C[6]$ :

G. Gentzen's cut-elimination theorem holds in GLC. That is, if a sequent in $G L C$ is provable, then it is also provable without cut.

It should be interesting and important to know whether or not the conjecture is affirmative. But it is remained open up to now.

Our theorem implies that a special case of the conjecture is yes. That is, if a sequent in $L K$ is provable in $G L C$, then by our theorem it should be also provable in $L K$. Therefore it should be provable without cut by Gentzen's cut-elimination theorem [5].

This paper is divided into three chapters, each of which contains one of our proofs of the theorem. They are written by S. Seki, S. Maehara and T. Nishimura respectively.

\section{Chapter I. The First Proof (by S. SEkI)}

\section{§1. $T L K$.}

At first, we shall formulate a type-logic in Gentzen's style. The symbols which occur in the system are divided into logical symbols and variables. The logical symbols are the negation sign 7 , the implication sign $\supset$, the disjunction sign $\vee$, the conjunction sign $\wedge$, the universal quantifier $\mathrm{V}$ and the existential quantifier $\mathrm{H}$.

The variables are divided into free variables and bound variables. To each of them a non-negative integer is associated and said to be its type. Semantically, the variables of type 0 , type 1 , type $2, \ldots$ are treated respectively as ranging over a domain of individuals, a domain of predicates, a domain of predicates of predicates and so on.

The free variables of type $\tau$ are denoted by

$$
a^{\tau}, b^{\tau}, \cdots, a_{1}^{\tau}, a_{2}^{\tau}, \cdots
$$

and the bound variables of type $\tau$ are denoted by

$$
\begin{aligned}
x^{\tau}, y^{\tau}, \cdots, x_{1}{ }^{\tau}, x_{2}{ }^{\tau}, \cdots \\
-270-
\end{aligned}
$$


In this chapter we shall use only the variables having one argumentplace. And we shall use no function-symbols. But we can treat also the case where those symbols occur in the same way.

Among expressions (i. e. finite concatenations of symbols) we distinguish formulas. The simplest, so-called pirmitive formulas are expressions of the form:

$$
a^{\tau+1} b^{\tau}
$$

Formulas are built from simpler ones by means of logical symbols. For example, the following expressions are formulas :

$$
\forall x^{5}\left(a^{6} x^{5} \vee>a^{6} x^{5}\right), \quad a^{2} b^{1} \supset \xi y^{2}\left(y^{2} b^{1}\right) .
$$

A formula containing a variable $a^{\tau}$ is often denoted by $A\left(a^{\tau}\right)$. An expression obtained from $A\left(a^{\tau}\right)$ by replacing $a^{\tau}$ by the symbol $*$ is called a variety of type $\tau+1$. A free variables of type 0 is called a variety of type 0 .

Let $B\left(b^{\tau}\right)$ be a formula. If we replace $b^{\tau}$ in $B\left(b^{\tau}\right)$ by a variety $V$ of type $\tau$ in the usual way, then we obtain a formula, which is denoted by $B(V)$.

We shall denote finite sequences of formulas by

$$
\Gamma, \Delta, \Theta, \cdots \text {. }
$$

These sequences may be empty. Expressions of the form

$$
\Gamma \rightarrow \Delta
$$

are called sequents. Proof-figures are defined in the same way as in Gentzen's $L K$. However the inference-shemata with respect to the quantifiers should be modified as follows :

$$
\frac{A(V), \Gamma \rightarrow \Delta}{\forall x^{\tau} A\left(x^{\tau}\right), \Gamma \rightarrow \Delta} \quad \frac{\Gamma \rightarrow \Delta, A\left(a^{\tau}\right):}{\Gamma \rightarrow \Delta, \forall x^{\tau} A\left(x^{\tau}\right)}
$$

$\left(\begin{array}{l}V \text { may be an arbitrary } \\ \text { variety of type } \tau \text {. }\end{array}\right) \quad\left(\begin{array}{l}a^{\tau} \text { may not be contained } \\ \text { in the lower sequent. }\end{array}\right)$

$$
\frac{A\left(a^{\tau}\right), \Gamma \rightarrow \Delta}{\text { H } x^{\tau} A\left(x^{\tau}\right), \Gamma \rightarrow \Delta} \quad \frac{\Gamma \rightarrow \Delta, A(V)}{\Gamma \rightarrow \Delta, \text { 芩 } x^{\tau} A\left(x^{\tau}\right)}
$$

$\left(\begin{array}{l}a^{\tau} \text { may not be contained } \\ \text { in the lower sequent. }\end{array}\right) \quad\left(\begin{array}{l}V \text { may be an arbitrary } \\ \text { variety of type } \tau .\end{array}\right)$

We shall call this system of logic $T L K$. Of course, $T L K$ is an extension 
of Gentzen's $L K$.

§2. Logical Truth.

Let $U$ be a non-empty set. We shall define a sequence of sets recursively as follows :

$$
\left\{\begin{array}{l}
U_{0}=U \\
U_{n+1}=2^{U n}(\text { power set) }
\end{array}\right.
$$

We shall call the set

$$
\bar{U}=\bigcup_{n=0}^{\infty} U_{n}
$$

the closure of $U$, and elements of $\bar{U}$ the mathematical entities with respect to $U$.

Let $A$ be a formula (in $T L K$ ). We assume that all the free variables contained in $A$ have been arranged as follows:

$$
\text { (1) } a_{1}^{\tau_{1}}, a_{2}^{\tau_{2}}, \cdots, a_{n}^{\tau_{n}} \text {. }
$$

We consider a system $R$ formed by a non-empty set $U$, and by a sequence

$$
E_{1}, E_{2}, \cdots, E_{n}
$$

of certain mathematical entities with respect to $U$, with the same number of terms as the sequence (1). The mathematical nature of each $E_{i}$ depends on the logical character of the corresponding variable $a_{i}{ }^{\tau s}$. Thus, for example, if $\tau_{i}=0$, then $E_{i}$ should be an element of $U_{0}=U$. If $\tau_{i}=1$, then $E_{i}$ should be an element of $U_{1}=2^{\sigma}$, i. e. a subset of $U$; and so on. Such a system

$$
R=\left\{U ; E_{1}, E_{2}, \cdots, E_{n}\right\}
$$

is called a realization of $A$.

We assume it to be clear under what conditions a formula $A$ is said to hold in $R$. Roughly speaking, this means that $A$ turns to be true if

$(\alpha)$ all the bound variables of type $\tau$ occurring in $A$ are assumed to range over the set $U_{\tau}$,

$(\beta)$ the logical symbols are interpreted in the usual way,

( $\gamma$ ) each of $a_{i}{ }^{\tau}$ is understood to denote the corresponding entity $E_{i}$. A formula $A$ is said to be logically true if it holds in its every reali- 
zation. A sequent: $A_{1}, \cdots, A_{r} \rightarrow B_{1}, \cdots, B_{\delta}(r+s>0)$ is said to be logically true, if the formula

$$
>A_{1} \vee \cdots \vee>A_{r} \vee B_{1} \vee \cdots \vee B_{8}
$$

is logically true. The sequent $\rightarrow$ is said to be not logically true.

An important property of the notion of logical truth is stated in the following well-known theorem :

GODEL'S COMPLETENESS THEOREM. Let $S$ be:a sequent in LK. Then, $S$ is provable in $L K$, if and only if it is logically true.

\section{§3. Proof OF THE TheORem.}

Now we shall prove the theorem, which is stated in the preface of this paper.

In this section, we shall formulate the theorem as follows:

TheOREM. If a sequent in $L K$ is provable in $T L K$, then it is also provable in $L K$.

To prove this theorem, it is sufficient to prove the following

Lemma. If a sequent is provable in TLK, then it is logically true.

Assume that the lemma has been proved, and assume that a sequent $S$ in $L K$ is provable in $T L K$. Then, by the lemma, it should be logically true. Hence, by Gödel's completeness theorem, it should be provable in $L K$. The proof of the theorem is thus completed.

Proof of LEMMA. For a proof-figure $P$, we call the number of sequents contained in $P$ the length of $P$. Let $S$ be a sequent in $T L K$, and assume that $S$ is provable in TLK. The minimum of the lengths of proof-figures, whose end-sequents are $S$, is called the rank of $S$.

We shall prove the theorem by mathematical induction with respect to the rank of $S$.

BAsIs (Case where the rank is 1). In this case; the proof-figure for $S$ is $S$ itself, and $S$ should be of the form

$$
A \rightarrow A \text {. }
$$

Of course, the formula $7 A \vee A$ holds in its any realization by the law of excluded middle. 
InDUCTION STEP. Assume that the theorem is true when the rank is less than $m$, and assume that the rank of $S$ is $m$, and $P$ is the prooffigure for $S$ whose length is $m$. Let (I) be the lowest inference of $P$. Then, by the hypothesis of the induction, the theorem should hold for upper sequents in (I). Now, we shall consider the case where (I) is of the form:

$$
\frac{A, C_{1}, \cdots, C_{m} \rightarrow D_{1}, \cdots, D_{n}}{A \wedge B, C_{1}, \cdots, C_{m} \rightarrow D_{1}, \cdots, D_{n}}
$$

Let $R$ be a realization of the formula

(2) $>(A \wedge B) \vee>C_{1} \vee \cdots \vee>C_{m} \vee D_{1} \vee \cdots \vee D_{n}$

The formula

$$
>A \vee>C_{1} \vee \cdots \vee>C_{m} \vee D_{1} \vee \cdots \vee D_{n}
$$

should hold in $R$, because $R$ is also a realization of (3). If at least one of $>C_{1}, \cdots, \nabla C_{m}, D_{1}, \cdots, D_{n}$ holds in $R$, then clearly the formula (2) holds in $R$. Assume that none of $>C_{1}, \cdots,>C_{m}, D_{1}, \cdots, D_{n}$ holds in $R$. Since (3) holds in $R,>A$ should hold in $R$. Hence $\neg(A \wedge B)$ holds in $R$. This means that (2) holds in $R$. Thus, $S$ should be logically true.

Next, we shall consider the case where (I) is of the form:

$$
\frac{C_{1}, \cdots, C_{m} \rightarrow D_{1}, \cdots, D_{n}, A\left(a^{\tau}\right)}{C_{1}, \cdots, C_{m} \rightarrow D_{1}, \cdots, D_{n}, \forall x^{\tau} A\left(x^{\tau}\right)}
$$

Let $R$ be a realization of the formula

$$
\text { (4) }>C_{1} \vee \cdots \vee>C_{m} \vee D_{1} \vee \cdots \vee D_{n} \vee \forall x^{\tau} A\left(x^{\tau}\right)
$$

If at least one of $>C_{1}, \cdots,>C_{m}, D_{1}, \cdots, D_{n}$ holds in $R$, then clearly the formula (4) holds in $R$. Assume that none of $>C_{1}, \cdots,>C_{m}, D_{1}, \cdots, D_{n}$ holds in $R$. Let $R^{\prime}$ be a realization of

$$
\text { (5) }>C_{1} \vee \cdots \vee>C_{m} \vee D_{1} \vee \cdots \vee D_{n} \vee A\left(a^{r}\right) \text {, }
$$

and assume that $R^{\prime}$ is an extension of $R$. Since $S^{\prime}$ is logically true by the hypothesis of the induction, $A\left(a^{\tau}\right)$ should hold in $R^{\prime}$. Remembering that $R^{\prime}$ is an arbitrary extension of $R$, we see that (4) should hold in $R$. Therefore (4) should be logically true.

The cases where (I) is of another form can be treated similarly. Thus the lemma is proved. 
REMARK 1. For any ordinal number $\alpha$, we can construct a predicate calculus of $\alpha$ th order. Our theorem is also true for those systems.

REMARK 2. For any natural number $n>1$, we can construct a formula in the predicate calculus of $n$th order such that satisfies the following conditions :

(a) provable in the predicate calculus of $(n+1)$ th order,

(b) not provable in the predicate calculus of $n$th order.

\section{Chapter II. The Second Proof (by S. Maehara)}

In this chapter, we shall show that our theorem can be proved in almost the same way as in Rasiowa [2].

$\S 1$. We shall use two formal systems $\mathbf{S}$ and $\mathbf{S}_{0} . \mathbf{S}$ is a system for the predicate calculus of order $\omega$, and $\mathbf{S}_{0}$ is a system for the predicate calculus of first order.

As formulas, in $\mathbf{S}$ and in $\mathbf{S}_{0}$, we shall use ones explained in $\S 1$ of Chapter I, and no varieties nor sequents will be used.

1. Postulates for the formal system $\mathbf{S}$ :

1. 1. Axioms :

1) Identically true formulas, each of which is the result of substituting some formulas, in our sense, for propositional variables throughout a provable formula in the propositional calculus.

2) Formulas of the form

$$
\forall x^{\tau} A\left(x^{\tau}\right) \supset A\left(a^{\tau}\right), \quad A\left(a^{\tau}\right) \supset \text { H } x^{\tau} A\left(x^{\tau}\right)
$$

or

$$
\text { 炙 } x^{\tau+1} \forall y^{\tau}\left[\left(A\left(y^{\tau}\right) \supset x^{\tau+1} y^{\tau}\right) \wedge\left(x^{\tau+1} y^{\tau} \supset A\left(y^{\tau}\right)\right] .\right.
$$

1. 2. Rules of inference :

$$
\begin{aligned}
& -\frac{A \supset B}{B}, \\
& \frac{B \supset A\left(a^{\tau}\right)}{B \supset \forall x^{\tau} A\left(x^{\tau}\right)}, \quad \frac{A\left(a^{\tau}\right) \supset B}{\text { H } x^{\tau} A\left(x^{\tau}\right) \supset B}
\end{aligned}
$$


In each of the last two rules, the free variable $a^{\tau}$ shall not occur in $A\left(x^{\tau}\right)$ and in $B$.

2. The formal system $\mathbf{S}_{0}$ is distinguished from $\mathbf{S}$ by only the following RESTRICTION ON BOUND VARIABLES : Bound variables used in the system $\mathbf{S}_{0}$ are ones of type 0 only.

3. It is easily proved that the system $\mathbf{S}$ is equivalent to $T L K$ in the preceding chapter, and that $\mathbf{S}_{0}$ is essentially equivalent to the ordinary system for the predicate calculus of first order.

$\S 2$. Lindenbaum Algebra of $\mathbf{S}_{0}$. Let $\left|A_{0}\right|$ denote the set of all $\mathbf{S}_{0}$-formulas $B_{0}$ such that $\left(A_{0} \supset B_{0}\right) \wedge\left(B_{0} \supset A_{0}\right)$ is $\mathbf{S}_{0}$-provable. Then the Lindenbaum algebra $L_{0}$ of $\mathbf{S}_{0}$ is the Boolean lattice of all cosets $\left|A_{0}\right|$ with the operations

$$
\left|A_{0}\right| \cap\left|B_{0}\right|=\left|A_{0} \wedge B_{0}\right|,\left|A_{0}\right| \cup\left|B_{0}\right|=\left|A_{0} \vee B_{0}\right| \text { and }\left|A_{0}\right|^{c}=\left|>A_{0}\right|
$$

The following propositions are easy to prove.

Proposition 1. If $\left|A_{0}\right|=1$, where 1 is the unit element of $L_{0}$, then $A_{0}$ is $\mathbf{S}_{0}$-provable.

Proposition 2. Let $J_{0}$ be the set of all free variables of type 0 , then

$$
\left|\forall x^{0} A_{0}\left(x^{0}\right)\right|=\bigcap_{a^{0} \in J_{0}}\left|A_{0}\left(a^{0}\right)\right| \text { and }\left|\mathbb{H} x^{0} A_{0}\left(x^{0}\right)\right|=\bigcup_{a^{0} \in J_{0}}\left|A_{0}\left(a^{0}\right)\right| \text {. }
$$

$\S 3$. 1. Let $L$ be the Boolean lattice which is the minimal complete extention of $L_{0}$, and we define recursively the sequence of sets $\Phi_{0}, \Phi_{l}$, $\Phi_{2}, \cdots \cdots$ as follows :

$$
\left\{\begin{array}{c}
\Phi_{0}=J_{0}(=\text { the sets of all free variables of type } 0), \\
\Phi_{\tau+1}=L^{\Phi_{\tau}} \quad \text { (set-theoretic power set). }
\end{array}\right.
$$

2. For each free variable $a^{\tau}$, we define the element $a^{\tau *}$ of $\Phi_{\tau}$ recursively as follows :

$$
\begin{gathered}
a^{0 *}=a^{0} \\
a^{\tau+1 *}\left(\varphi^{\tau}\right)= \begin{cases}\left|a^{\tau+1} b^{\tau}\right|, & \text { if } \varphi^{\tau}=b^{\tau *} \\
0, & \text { otherwise }\end{cases}
\end{gathered}
$$

3. Let $F\left(a^{\tau}, b^{\sigma}, \cdots\right)$ be an arbitrary $\mathbf{S}$-formula containing no free variable except $a^{\tau}, b^{0}, \cdots$. Then, for that formula, we define the mapping 
$F^{*}\left(\varphi^{\tau}, \phi^{\sigma}, \cdots\right)$ from $\Phi_{\tau} \times \Phi_{\sigma} \times \cdots$ to $L$, recursively as follows :

1) Case where $F\left(a^{\tau+1}, b^{\tau}\right)$ is a formula of the form $a^{\tau+1} b^{\tau}$ :

$$
F^{*}\left(\varphi^{\tau+1}, \phi^{\tau}\right)=\varphi^{\tau+1}\left(\phi^{\tau}\right)
$$

2) Case where $F=>A, F=A \wedge B, F=A \vee B$ or $F=A \supset B$ :

$$
F^{*}=A^{* c}, F^{*}=A^{*} \cap B^{*} F^{*}=A^{*} \cup B^{*} \text { or } F^{*}=A^{* c} \cup B^{*},
$$

respectively.

3) Case where $F\left(b^{\sigma}, \cdots\right)=\forall x^{\tau} A\left(x^{\tau}, b^{\sigma}, \cdots\right)$ or $F\left(b^{\sigma}, \cdots\right)=\xi x^{\tau} A\left(x^{\tau}, b^{\tau}, \cdots\right)$ :

$$
F^{*}\left(\phi^{\sigma}, \cdots\right)=\bigcap_{\varphi^{\tau} \in \Phi_{\tau}} A^{*}\left(\varphi^{\tau}, \psi^{\sigma}, \cdots\right)
$$

or

$$
F^{*}\left(\phi^{\sigma}, \cdots\right)=\bigcup_{\varphi^{\tau} \epsilon \Phi_{\tau}} A^{*}\left(\varphi^{\tau}, \phi^{\sigma}, \cdots\right)
$$

respectively.

4. The following proposition is easily proved, by the help of Proposition 2 .

Proposition 3. If $F_{0}\left(a^{\tau}, b^{0}, \cdots\right)$ is an $\mathbf{S}_{0}$-formula containing no free variable except $a^{\tau}, b^{\sigma}, \cdots$, , then

$$
\left|F_{0}\left(a^{\tau}, b^{\sigma}, \cdots\right)\right|=F_{0}^{*}\left(a^{* *}, b^{a *}, \cdots\right)
$$

$\S 4$ Propositin 4. If an S-formula $F\left(a^{\tau}, b^{a}, \cdots\right)$, which contains no free variable except $a^{\tau}, b^{a}, \cdots$. is $\mathrm{S}-$ provable, then

$$
F^{*}=\left(\varphi^{\tau}, \phi^{\sigma}, \cdots\right)=1 \quad\left(\varphi^{\tau} \epsilon \Phi_{\tau}, \phi^{\sigma} \epsilon \Phi_{\sigma}, \cdots\right)
$$

Proof (inductive proof).

1) Case where $F$ is an identically true formula: The proposition evidently holds.

2) Case where $F$ has the form

$$
\begin{gathered}
\forall x^{\tau} A\left(x^{\tau}, b^{\sigma}, \cdots\right) \supset A\left(a^{\tau}, b^{\sigma}, \cdots\right): \\
F^{*}\left(\varphi^{\tau}, \phi^{\sigma}, \cdots\right)=\left[\bigcap_{\xi^{\tau} \in \psi_{\tau}} A^{*}\left(\xi^{\tau}, \phi^{\sigma}, \cdots\right)\right]^{c} \cup A^{*}\left(\varphi^{\tau}, \phi^{o}, \cdots\right) \\
\geqq A^{*}\left(\varphi^{\tau}, \phi^{\sigma}, \cdots\right)^{c} \cup A^{*}\left(\varphi^{\tau}, \phi^{\sigma}, \cdots\right)=1 ; \\
\therefore \quad F^{*}\left(\varphi^{\tau}, \psi^{\sigma}, \cdots\right)=1 .
\end{gathered}
$$

3) Case where $F\left(b^{\sigma}, \cdots\right)$ has the form

$$
\begin{gathered}
\exists x^{\tau+1} \forall y^{\tau}\left[\left(A\left(y^{\tau}, b^{\sigma}, \cdots\right) \supset x^{\tau+1} y^{\tau}\right) \wedge\left(x^{\tau+1} y^{\tau} \supset A\left(y^{\tau}, b^{\sigma}, \cdots\right)\right]:\right. \\
F^{*}\left(\phi^{\sigma}, \cdots\right)=\bigcup_{\xi^{\tau+1} \in \Phi_{\tau+1}} \bigcap_{\eta^{\tau} \epsilon \Phi_{\tau}}\left[\xi^{\tau+1}\left(\eta^{\tau}\right)+A^{*}\left(\eta^{\tau}, \phi^{\sigma}, \cdots\right)\right]^{c}, \\
-277-
\end{gathered}
$$


where + means the 'symmetric difference'. Hence,

$$
\begin{gathered}
F^{*}\left(\psi^{\sigma}, \cdots\right)=\bigcap_{\eta^{\tau} \in \Phi_{\tau}}\left[A^{*}\left(\eta^{\tau}, \phi^{\sigma}, \cdots\right)+A^{*}\left(\eta^{\tau}, \psi^{a}, \cdots\right)\right]^{c}=1 ; \\
\therefore \quad F^{*}\left(\psi^{\sigma}, \cdots\right)=1 .
\end{gathered}
$$

4) Case where $F$ is $B\left(a^{\tau}, b^{\sigma} \cdots\right)$, and $A\left(a^{\tau}, b^{\sigma}, \cdots.\right)$ and $A\left(a^{\tau}, b^{\alpha}, \cdots.\right) \supset$ $B\left(a^{\tau}, b^{0}, \cdots\right)$ are both S-provable :

By the hypothesis of induction,

$$
A^{*}\left(\varphi^{\tau}, \phi^{\sigma}, \cdots\right)=1
$$

and

$$
\begin{gathered}
A^{*}\left(\varphi^{\tau}, \psi^{\sigma}, \cdots\right)^{c} \cup B^{*}\left(\varphi^{\tau}, \phi^{\sigma}, \cdots\right)=1 ; \\
\therefore \quad F^{*}=B^{*}\left(\varphi^{\tau}, \psi^{\sigma}, \cdots\right)=1 .
\end{gathered}
$$

5) Case where $F$ is $B\left(b^{\alpha}, \cdots\right) \supset \forall x^{\tau} A\left(x^{\tau}, b^{\sigma}, \cdots\right), B\left(b^{\sigma}, \cdots\right) \supset A\left(a^{\tau}, b^{a}, \cdots\right)$ is S-provable, and $a^{\tau}$ occurs in neither $A\left(x^{\tau}, b^{\alpha}, \cdots\right)$ nor $B\left(b^{a}, \cdots\right)$ :

By the hypothesis of induction,

$$
\begin{gathered}
B^{*}\left(\phi^{\sigma}, \cdots\right)^{c} \cup A^{*}\left(\varphi^{\tau}, \psi^{\sigma}, \cdots\right)=1 ; \\
\therefore \quad F^{*}=B^{*}\left(\phi^{\sigma}, \cdots\right)^{c} \cup\left[\bigcap_{\varphi^{\tau} \in \Phi_{r}} A^{*}\left(\varphi^{\tau}, \psi^{\sigma}, \cdots\right)=1 .\right.
\end{gathered}
$$

6) Case where $F$ is $\mathbb{H} x^{\tau} A\left(x^{\tau}, b^{\sigma}, \cdots\right) \supset B\left(b^{a}, \cdots\right), A\left(a^{\tau}, b^{a}, \cdots\right) \supset B\left(b^{\sigma}, \cdots\right)$ is $\mathbf{S}$-provable, and $a^{\tau}$ occurs in neither $A\left(x^{\tau}, b^{q}, \cdots\right)$ nor $B\left(b^{\alpha}, \cdots\right)$ : The treatment is similar to 5), q. e. d.

§5. By Propositions 3, 4 and 1, we have the following

Theorem. If an $\mathbf{S}_{0}$-formula is $\mathbf{S}$-provable, then the $\mathbf{S}_{0}$-formula is $\mathbf{S}_{0}-$ provable.

Chapter III. The Third Proof and an Extension of the Theorem (by T. Nishimura)

In this chapter we give a non-constructive consistency-proof of the theory of real numbers. The applied system is obtained by generalizing the system $Z$ given by $\mathrm{K}$. Schütte [3]. The system is denoted by $G Z$.

In $\S 1$ we formulate $G Z$ into $G$. Gentzen's style.

In $\S 2$ we give a system equivalent to $G$. Takeuti's $G^{1} L C$ and we 
show that its super-arithmetical proof-figure is transformed to a prooffigure in $G Z$. Here a super-arithmetical proof-figure means a proof-figure which contains no predicates except $=$ and no functions except ${ }^{\prime},+$ and $\cdot$

In $\S 3$ we give some important meta-theorems on $G Z$.

In $\S 4$ we give the consistency-proof of the theory of real numbers and a theorem on G. Takeuti's fundamental conjecture for his $G^{1} L C$.

\section{$\S 1$. The System $G Z$.}

In this section we formulate the system $G Z$.

1. Symbols.

We apply the following fundamental symbols: Symbol 0, function symbols ',,$+ \cdot$, bound variables $x, y, z$ etc., particular variety symbols with $m$ arguments $V_{\tau}, V_{\xi}, V_{\eta}$ etc., (where $\tau, \xi, \eta$ etc. are arbitrary sets of $m$-tuples of natural numbers), bound $V$-variables with $m$ arguments $\varphi$, $\phi$ etc., symbol $\lambda$, logical symbols $\wedge, \neg, \forall$ and symbol $\rightarrow$.

The system $G Z$ contains symbols of power of the continuum.

If necessary we apply several letters for abbreviation.

2. Terms are constructed as follows :

(1) the symbol 0 is a term; (2) if $t$ is a term, so is $t^{\prime}$, and if $t_{1}$ and $t_{2}$ are terms, so are $t_{1}+t_{2}$ and $t_{1} \cdot t_{2}$. Particularly figures of the form 0 , $0^{\prime}, 0^{\prime \prime}, \cdots$ are called numerals. For an term $t$ we can decide its numerical value by the well-known way. When $n$ is a natural number, $0^{n+\cdots \prime}$ is called the numeral expressing $n$ and denoted by $n$.

3. Varieties and formulas are constructed as follows.

(1) Particular varieties with $m$ arguments are varieties with $m$ arguments.

(2) If $t_{1}$ and $t_{2}$ are terms, then $t_{1}=t_{2}$ is a formula. If $V$ is a variety with $m$ arguments and $t_{1}, \cdots, t_{m}$ are terms, then $[V]\left(t_{1}, \cdots, t_{m}\right)$ is a formula.

(3) If $A$ is a formula, so is $7 A$.

(4) If $A$ and $B$ are formulas, so is $A \wedge B$. 
(5) If $F(t)$ is a formula, so is $\forall x F(x)$. If $F\left(t_{1}, \cdots, t_{m}\right)$ is a formula, then $\lambda x_{1} \cdots x_{m} F\left(x_{1}, \cdots, x_{m}\right)$ is a variety with $m$ arguments.

(6) If $F\left(V_{\tau}\right)$ is a formula and $V_{\tau}$ is a particular variety with $m$ arguments, then $\forall \varphi F(\varphi)$ is a formula, where $\varphi$ is a bound $V$-variable with $m$ arguments.

4. We call a figure of the following form a sequent,

$$
A_{1}, \cdots, A_{\mu} \rightarrow B_{1}, \cdots, B_{\nu}
$$

where $A_{1}, \cdots, A_{\mu}, B_{1}, \cdots, B_{\nu}$ are arbitrary formulas. And it may happen that $\mu=0$ or $\nu=0$. We say that $A_{1}, \cdots, A_{\mu}$ are in the antecedent and $B_{1}, \cdots, B_{\nu}$ are in the succedent.

5. Sequents of the following forms are called beginning sequents:

(1) a sequent of the form $\rightarrow P$, where $P$ is a ture formula of the form $t_{1}=t_{2}$,

(2) a sequent of the form $P \rightarrow$, where $P$ is a false formula of the form $t_{1}=t_{2}$,

(3) a sequent of the form $\rightarrow\left[V_{\tau}\right]\left(t_{1}, \cdots, t_{m}\right)$, where $V_{\tau}$ is a particular variety with $m$ arguments and $t_{1}, \cdots, t_{m}$ are terms numerical values of which are $n_{1}, \cdots, n_{m}$ and the $m$-tuple of natural numbers $n_{1}, \cdots, n_{m}$ belongs to $\tau$,

(4) a sequent of the form $\left[V_{\tau}\right]\left(t_{1}, \cdots t_{m}\right) \rightarrow$, where $V_{\tau}$ is a particular variety with $m$ arguments and $t_{1}, \cdots, t_{m}$ are terms numerical values of which are $n_{1}, \cdots, n_{m}$ and the $m$-tuple of natural numbers $n_{1}, \cdots, n_{m}$ does not belong to $\tau$,

(5) $D \rightarrow D$, where $D$ is an arbitrary formula.

6. RULES OF INFERENCE.

If $S_{1}, \cdots, S_{m}$ and $S$ are sequents, then a figure of the form

$$
\frac{S_{1} \cdots S_{m}}{S}
$$

is called a rule of inference. $S_{1}, \cdots, S_{m}$ are called the upper sequents and $S$ is called the lower sequent of the rule of inference. In our case $G Z$ contains the following rules of inference.

In what follows, capita1 Greek letters $\Gamma, \Pi$ etc. express finite sequences 
of formulas.

(1) Structural rules of inference:

Thinning in antecedent

$$
\frac{\Gamma \rightarrow \Delta}{D, \Gamma \rightarrow \Delta}
$$

Interchange in antecedent

$$
\frac{\Gamma, D, C, \Pi \rightarrow \Delta}{\Gamma, C, D, \Pi \rightarrow \Delta}
$$

Contraction in antecedent

$$
\frac{D, D, \Gamma \rightarrow \Delta}{D, \Gamma \rightarrow \Delta}
$$

Thinning in succedent

$$
\frac{\Gamma \rightarrow \Delta}{\Gamma \rightarrow \Delta, D}
$$

Interchange in succedent

$$
\frac{\Gamma \rightarrow \Delta, D, C, \Lambda}{\Gamma \rightarrow \Delta, C, D, \Lambda}
$$

Contraction in succedent

$$
\frac{\Gamma \rightarrow \Delta, D, D}{\Gamma \rightarrow \Delta, D}
$$

where $C$ and $D$ are arbitrary formulas called principal formulas of each rule of inference.

(2) Logical rules of inference:

$\wedge$-in antecedent

$$
\frac{A, \Gamma \rightarrow \Delta}{A \wedge B, \Gamma \rightarrow \Delta}, \quad \frac{B, \Gamma \rightarrow \Delta}{A \wedge B, \Gamma \rightarrow \Delta}
$$

$>$-in antecedent

$$
\frac{\Gamma \rightarrow \Delta, A}{>A, \Gamma \rightarrow \Delta}
$$

$\wedge$-in succedent

$$
\frac{\Gamma \rightarrow \Delta, A \quad \Gamma \rightarrow \Delta, B}{\Gamma \rightarrow \Delta, A \wedge B}
$$

7 -in succedent

$$
\frac{A, \Gamma \rightarrow \Delta}{\Gamma \rightarrow \Delta,>A}
$$

where $A$ and $B$ are arbitrary formulas called side-formulas of each rule of inference. $A \wedge B$ or $>A$ is called principal formula of each rule of inference.

$\forall$ on individual-in antecedent

$$
\frac{F(t), \Gamma \rightarrow \Delta}{\forall x F(x), \Gamma \rightarrow \Delta} \text {, where } t \text { is an arbitrary term. }
$$

$\mathrm{V}$ on individual-in succedent

$$
\frac{\Gamma \rightarrow \Delta, F(n) \text { for every numeral } n}{\Gamma \rightarrow \Delta, \forall x F(x)}
$$

This is called the infinite induction. $F(n)$ is called the side-formula and $\mathrm{V} x F(x)$ is called the principal formula of each rule of inference.

$\forall$ on variety-in antecedent 


$$
\frac{F(V), \Gamma \rightarrow \Delta}{\forall \varphi F(\varphi), \Gamma \rightarrow \Delta} \text {, where } V \text { is an arbitrary variety. }
$$

$\forall$ on variety-in succedent

$$
\frac{\Gamma \rightarrow \Delta, F\left(V_{\tau}\right) \text { for every particular variety } V_{\tau}}{\Gamma \rightarrow \Delta, \forall \varphi F(\varphi)}
$$

This is called the infinite induction on variety.

(3) Rules of inference with respect to the symbol $\lambda$ :

in antecendent

in succedent

$$
\begin{aligned}
& \frac{A\left(t_{1}, \cdots, t_{m}\right), \Gamma \rightarrow \Delta}{\left[\lambda x_{1} \cdots x_{m} A\left(x_{1}, \cdots, x_{m}\right)\right]\left(t_{1}, \cdots, t_{m}\right), \Gamma \rightarrow \Delta}, \\
& \frac{\Gamma \rightarrow \Delta, A\left(t_{1}, \cdots, t_{m}\right)}{\Gamma \rightarrow \Delta,\left[\lambda x_{1} \cdots x_{m} A\left(x_{1}, \cdots, x_{m}\right)\right]\left(t_{1}, \cdots, t_{m}\right)}
\end{aligned}
$$

(4) Cut

$$
\frac{\Gamma \rightarrow \Delta, D \quad D, \Pi \rightarrow \Lambda}{\Gamma, \Pi \rightarrow \Delta, \Lambda}
$$

where $D$ is an arbitrary formula called the cut-formula.

7. The number of logical symbols and $\lambda$ 's of a formula is called the degree of the formula. The degree of a cut is the degree of the cut-formula.

8. We introduce a concept "proof-figure" into the system. Under a proof-figure we understand a figure of finite or infinite sequents, built up in the following manner: uppermost sequents are always beginning sequent; every sequent is a lower sequent of at most one rule of inference ; and every sequent, except just one, the end-sequent, is upper sequent of just one rule of inference. To every sequent of proof-figure corresponds an ordinal number of the third class as follows. (1) The ordinal number of a beginning sequent is zero. (2) The ordinal number of the lower sequent of a structural rule of inference is equal to that of the upper sequent. (3) The ordinal number of the lower sequent of a cut, a logical rule of inference or a rule of inference with respect to the symbol $\lambda$ is greater than those of upper sequents.

A proof-figure in $G Z$ is called a $G Z$-proof-figure. If we have a $G Z$ - 
proof-figure to a sequent $\Gamma \rightarrow \Delta$, then we say that $\Gamma \rightarrow \Delta$ is $G Z$-provable.

9. Logical system $P G Z$ and $F P G Z$. :

The system $P G Z$ is obtained by modifying $G Z$ as follows: in logical rules of inference of $G Z, \forall$ on variety-in antecedent, we restrict $V$ to particular variety. A proof-figure in $P G Z$ is called a $P$-proof-figure. A sequent to be provable in $P G Z$ is said to be $P$-provable.

A proof-figure of the system $F P G Z$ is a proof-figure of $P G Z$ the order of which is finite and which has the maximum degree of cuts. A prooffigure of $F P G Z$ is called a $F$-proof-figure. A sequent to be provable in $F P G Z$ is said to be $F$-provable.

§ 2. G. Takeuti's $G^{1} L C$.

1. By the following modification of $G Z$ we obtain the system equivalent to $\mathrm{G}$. 'Takeuti's $G^{1} L C$.

(1) As fundamental symbols, we add free variables $a, b, c$ etc. and free $V$-variables with $m$ arguments $\alpha, \beta, \gamma$ etc., $m=1,2,3, \cdots$.

Moreover we add function symbols with $m$ arguments $f$; $h$, etc. $(m=1$, $2, \cdots)$ and predicate symbols with $m$ arguments $p, q$ etc. $(m=1,2, \cdots)$.

(2) To the costruction rules of terms we add 'if $f$ is a function symbol with $\mathrm{m}$ arguments and if $t_{1}, \cdots, t_{m}$ are terms, then $f\left(t_{1}, \cdots, t_{m}\right)$ is a term.' And to the construction rules of formulas we add 'if $p$ is a predicate symbol with $m$ arguments and if $t_{1}, \cdots, t_{m}$ are terms, then $p\left(t_{1}, \cdots, t_{m}\right)$ is a formula.

(3) The logical rule of inference $\forall$ on individual-in succedent of $G Z$ is omitted, and we add $\forall$ on individual-in succedent of $G^{1} L C$ of the form

$$
\frac{\Gamma \rightarrow \Delta, F(a)}{\Gamma \rightarrow \Delta, \forall x F(x)}
$$

where $a$ is a free variable to be not contained in the lower sequent.

(4) The logical rules of inference $V$ on variety-in succedent are omitted, and we add $\mathrm{V}$ on variety of $G^{1} L C$ of the form 


$$
\frac{\Gamma \rightarrow \Delta, F(\alpha)}{\Gamma \rightarrow \Delta, \forall \varphi F(\varphi)}
$$

where $\alpha$ is a free $V$-variable to be not contained in the lower sequent.

(5) Moreover we add a rule of inference (inference of complete induction) to the system, which is of the form

$$
C I \frac{F(a), \Gamma \rightarrow \Delta, F\left(a^{\prime}\right)}{F(0), \Gamma \rightarrow \Delta, F(t)}
$$

where $a$ is a free variable to be not contained in the lower sequent and $t$ is an arbitrary term.

(6) Beginning sequents of $G Z$ are omitted except those of the form $D \rightarrow D$.

In what follows, we call our system the system $G^{1} L C$ with complete induction or briefly $G^{1} L C$, which contains the rule of inference $C I$.

2. We call a formula a super-arithmetical formula, which contains no predicates except $=$ and no functions except',,$+ \cdot$. A sequent $A_{1}, \cdots, A_{\mu}$ $\rightarrow B_{1}, \cdots, B_{\nu}$ is a super-arithmetical sequent, if $A_{1}, \cdots, A_{\mu}, B_{1}, \cdots, B_{\nu}$ are super-arithmetical. A proof-figure is called a super-arithmetical proof-figure, if it contains only super-arithmetical sequent.

A sequent $A_{1}, \cdots, A_{\mu} \rightarrow B_{1}, \cdots, B_{\nu}$ is called a simple sequent, if $A_{i}$ ( $i=1, \cdots$, $\mu$ ) are super-arithmetical and of the forms

$$
\forall x_{1} \cdots \forall x_{l} F\left(x_{1}, \cdots, x_{l}\right)
$$

where $F\left(a_{1}, \cdots, a_{l}\right)$ contains no quantifiers, and if $B_{j}(j=1, \cdots, \nu)$ are super-arithmetical and of the forms

$$
\neg \forall y_{1} \cdots \forall y_{m} H\left(y_{1}, \cdots, y_{m}\right)
$$

where $H\left(b_{1}, \cdots, b_{m}\right)$ contains no quatifiers.

It is well known that the theory of real numbers is derived from axioms $\Gamma_{0}$ in the system $G^{1} L C$ with complete induction restricted to super-arithmetical proof-figures, where $\Gamma_{0}$ are following axioms:

$$
\begin{aligned}
& \forall x \forall y\left(x^{\prime}=y^{\prime} \supset x=y\right) \\
& \forall x \neg\left(x^{\prime}=0\right) \\
& \forall x \forall y \forall z(x=y \supset(y=z \supset x=z)) \\
& \forall x \forall y\left(x=y \supset x^{\prime}=y^{\prime}\right) \\
& -284-
\end{aligned}
$$




$$
\begin{aligned}
& \forall x(x+0=x) \\
& \forall x \forall y\left(x+y^{\prime}=(x+y)^{\prime}\right) \\
& \forall x(x \cdot 0=0) \\
& \forall x \forall y\left(x \cdot y^{\prime}=x \cdot y+x\right)
\end{aligned}
$$

Here $A \supset B$ is the abbreviation of $\supset(A \wedge>B)$.

3. We transform any super-arithmetical proof-figure of the system $G^{1} L C$ with complete induction to a $G Z$-proof-figure. In what follows, if $\Gamma$ is of the form $A_{1}\left(\alpha_{1}, \cdots, \alpha_{l}, a_{1}, \cdots, a_{m}\right), \cdots, A_{\mu}\left(\alpha_{1}, \cdots, \alpha_{l}, a_{1}, \cdots, a_{m}\right)$, then $A_{1}\left(V_{1}, \cdots, V_{l}, t_{1}, \cdots, t_{m}\right), \cdots, A_{\mu}\left(V_{1}, \cdots, V_{l}, t_{1}, \cdots, t_{m}\right)$ is expressed by $\Gamma\left(V_{1}\right.$, $\left.\cdots, V_{l}, t_{1}, \cdots, t_{m}\right)$.

3. 1 THEOREM. Let a sequent $\Gamma\left(\alpha_{1}, \cdots, \alpha_{l}, a_{1}, \cdots, a_{m}\right) \rightarrow \Delta\left(\alpha_{1}, \cdots, \alpha_{l}, a_{1}, \cdots\right.$, $\left.a_{m}\right)$ be super-arithmetical and $G^{1} L C$-povable. Then for every l-tuple of particular varieties $V_{1}, \cdots, V_{l}$ and every $m$-tuple of numerals $n_{1}, \cdots, n_{m}$, the sequent

$$
\Gamma\left(V_{1}, \cdots, V_{l}, n_{1}, \cdots, n_{m}\right) \rightarrow \Delta\left(V_{1}, \cdots, V_{l}, n_{1}, \cdots, n_{m}\right)
$$

is $G Z$-provable by a smaller order than $\omega^{2}$.

PROoF is carried out in similar way as author's proof of Theorem 3 of $\S 3$ in [7].

\section{§3. Preliminary Theorems.}

In this section we discuss about the relations between systems $G Z$, $P G Z$ and $F P G Z$. First we easily see that an $F$-proof-figure is a $P$-prooffigure and a $P$-proof-figure is a $G Z$-proof-figure.

By the Theorems 2 and 6 in this section we see that these three systems are mutually equivalent.

1. TheOREm. (Elimination theorem of cut for FPGZ). If a sequent $\Gamma \rightarrow \Delta$ is $F$-provable, then we have an F-proof-figure without cut to $\Gamma \rightarrow \Delta$.

$\mathrm{P}_{\mathrm{ROOF}}$ is carried out in the same way as $\mathrm{K}$. Schütte's proof in his cut-elimination theorem for $Z$-proof-figure with finite order.

2. THEOREM (Elimination theorem of cut for $P G Z$ ). If a sequent $\Gamma \rightarrow$ $\Delta$ is $P$-provable, then we have an $F$-proof-figure without cut to $\Gamma \rightarrow \Delta$

PRoof is carried out in the same way as author's proof in Theorem 
7 of $\S 2$ in his paper [7].

3. Theorem (Decidability theorem). If $A$ is an arbitrary formula, then either $A$ or $7 A$ is $F$-provable without cut.

PROoF is carried out by the same way or anthor's proof in Theorem 8 of $\S 2$ in his paper [7].

4. LeMм. If $V$ is an arbitrary variety with $m$ arguments, then we have a particular variety $V_{\tau}$ with $m$ arguments such that the sequent

$$
\rightarrow \forall x_{1} \cdots \forall x_{m}\left\{[V]\left(x_{1}, \cdots, x_{m}\right) \sim\left[V_{\tau}\right]\left(x_{1}, \cdots, x_{m}\right)\right\}
$$

is $F$-provable without cut, where $A \sim B$ is the abbreviation of

$$
(A \supset B) \wedge(B \supset A) \text {. }
$$

Proof. If $V$ is a particular variety, then it is clear. Let $V$ be of the form $\lambda y_{1} \cdots y_{m} A\left(y_{1}, \cdots, y_{m}\right)$. Then for every $m$-tuple of natural numbers $n_{1}, \cdots, n_{m}$ either the formula $A\left(n_{1}, \cdots, n_{m}\right)$ or $7 A\left(n_{1}, \cdots, n_{m}\right)$ is $F$-provable without cut. Let $V_{\tau}$ be the set of $m$-tuples of natural number $n_{1}, \cdots, n_{m}$ such that $A\left(n_{1}, \cdots, n_{m}\right)$ is $F$-provable. Then the sequent

$$
\left.\rightarrow \forall x_{1} \cdots \forall x_{m} \mid[V]\left(x_{1}, \cdots, x_{m}\right) \sim\left[V_{\tau}\right]\left(x_{1}, \cdots, x_{m}\right)\right\}
$$

is $F$-provable without cut, q. e. d.

5. Lemma. If $A\left(n_{1}, \cdots, n_{m}\right)$ and $B\left(n_{1}, \cdots, n_{m}\right)$ are arbitrary formulas and

$$
\rightarrow \forall x_{1} \cdots \forall x_{m}\left\{A\left(x_{1}, \cdots, x_{m}\right) \sim B\left(x_{1}, \cdots, x_{m}\right)\right\}
$$

is $F$-provable without cut, then the sequents $F(A) \rightarrow F(B)$ and $F(B) \rightarrow F(A)$ are F-provable without cut.

Proof. We can easily prove by induction on the number of logical symbols in $F\left(V_{r}\right)$.

6. THEOREM. If a sequent $\Gamma \rightarrow \Delta$ is GZ-provable, then it is F-provable without cut.

Proof. We prove by transfinite induction on the order of the prooffigure to $\Gamma \rightarrow \Delta$.

If the order is zero, then it is clear.

If the order is not zero and the last rule of inference of the prooffigure is a structural rule of inference, then it is clear. If the last rule of inference is a logical rule of inference or a cut, then we have nine cases : $\wedge$-in antecedent, $\wedge$-in succedent, $>$-in antecedent, $>$-in succedent, 
$\forall$ on individual-in antecedent, $\forall$ on individual-in succedent, $\forall$ on variety-in antecedent, $\forall$ on variety-in succedent and cut. In cases that the last rule of inference is neither $\forall$ on variety in antecedent nor cut, we add the rule of inference to the $F$-proof-figure without cut to the upper sequent where is such $F$-proof-figure by the assumption of the transfinite induction. In case that the last rule of inference is a cut, we add the cut to the $F$-proof-figures without cut to the upper sequents, where are such $F$-proof-figures by the assumption of the transfinite induction, and we eliminate the new cut by Theorem 1 in this section. Let the last rule of inference be $\forall$ on variety-in antecedent and of the form

$$
\frac{F(V), \Gamma \rightarrow \Delta}{\forall \varphi F(\varphi), \Gamma \rightarrow \Delta}
$$

where $V$ is a variety. By Lemmas 4 and 5 we have a particular variety $V_{\tau}$ such that the sequent

$$
F\left(V_{\tau}\right) \rightarrow F(V)
$$

is $F$-provable. By the assumption of the transfinite induction we have an $F$-proof-figure to $F(V), \Gamma \rightarrow \Delta$. Therefore we have an $F$-proof-figure to $F\left(V_{\tau}\right), \Gamma \rightarrow \Delta$ by the cut with $F\left(V_{\tau}\right) \rightarrow F(V)$. And so we have an $F-$ proof-figure without cut to $\forall \varphi F(\varphi), \Gamma \rightarrow \Delta$ by Theorem 1 in this section.

\section{$\S 4$. Main Theorems.}

In this section we prove two main theorems.

1. MAIN THEOREM. The system $G Z$ is consistent.

Proof. If the sequent ' $\rightarrow$ ' is $G Z$-provable, then it is $F$-provable by Theorem 6 in $\S 3$. The sequent ' $\rightarrow$ ', however, is not $F$-provable by Theorem 1 in $\S 3$, q. e. d.

In section 2 we showed that $G^{1} L C$ with complete induction is included in $G Z$. Therefore we can see that $G Z$ contains the theory of real numbers. Hence from Main theorem 1 we can see that the theory of real numbers is consistent.

2. MAIN THEOREM. If a super-arithmetical simple sequent $\Gamma_{0}, \Gamma \rightarrow \Delta$ 
without free variable and free $V$-variable is $G^{1} L C$-provable, then we have a $L K$-proof figure without cut to $\Gamma_{0}, \Gamma \rightarrow \Delta$.

Proof. If we have a proof-figure in $G^{1} L C$ with complete induction to $\Gamma_{0}, \Gamma \rightarrow \Delta$, then we have a $G Z$-proof-figure to $\Gamma_{0}, \Gamma \rightarrow \Delta$ by Theorem 2 in $\S 2$. Then we have an $F$-proof-figure without cut to $\Gamma_{0}, \Gamma \rightarrow \Delta$ from Theorem 6 in $\S 3$. Because $\Gamma_{0}, \Gamma \rightarrow \Delta$ is simple, the proof-figure contains neither infinite induction nor $\forall$ on variety. Mereover it contains neither beginning sequents of the form $5,(3),(4)$ and (5) in $\S 1$. However beginning sequents of the form 5 (1) and 5 (2) are $L K$-provable from $\Gamma_{0}$. Therefore we obtain the theorem, q. e. d.

\section{References}

[1] K. Göde1, Die Vollständigkeit der Axiome des logischen Funktionenka1küls. Monatsh. f. Math. Phys., 37 (1930), 349-360.

[2] H. Rasiowa, On the $\varepsilon$-theorems. Fund. Math., 43 (1956) 156-165.

[3] K. Schütte, Beweistheoretische Erfassung der unendlichen Induktion in der Zahlentheorie. Math. Ann., 122 (1951), 369-389.

[4] G. Gentzen, Die Widerspruchsfreiheit der reinen Zahlentheorie. Math. Ann., 112 (1936), 493-565.

[5] G. Gentzen, Untersuchungen über das logische Schliessen. Math. Z., 39 (1934-5), 176-210, 405-431.

[6] G. Takeuti, On a generalized logic calculus. Jap. J. Math., 23 (1953), 3996. Errata to 'On a generalized logic calculus', Ibid., 24 (1954), 149-156.

[7] T. Nishimura, On a certain system with infinite induction. J. Math. Soc. Japan, to apper. 